OPEN

SUBJECT AREAS:

CHEMISTRY

MATERIALS SCIENCE

NANOSCIENCE AND

TECHNOLOGY

PHYSICS

Received

22 February 2013

Accepted

8 April 2013

Published

23 April 2013

Correspondence and requests for materials should be addressed to

U.S. (udo.

schwingenschlogl@

kaust.edu.sa)

\section{Molecular distortion and charge transfer effects in $\mathrm{ZnPc} / \mathrm{Cu}\left(\begin{array}{lll}1 & 1 & 1\end{array}\right)$}

\author{
B. Amin, S. Nazir \& U. Schwingenschlögl
}

KAUST, Physical Science \& Engineering Division, Thuwal 23955-6900, Kingdom of Saudi Arabia.

The adsorption geometry and electronic properties of a zinc-phthalocyanine molecule on a $\mathrm{Cu}(111)$ substrate are studied by density functional theory. In agreement with experiment, we find remarkable distortions of the molecule, mainly as the central $\mathrm{Zn}$ atom tends towards the substrate to minimize the $\mathrm{Zn}$-Cu distance. As a consequence, the $\mathrm{Zn}-\mathrm{N}$ chemical bonding and energy levels of the molecule are significantly modified. However, charge transfer induces metallic states on the molecule and therefore is more important for the $\mathrm{ZnPc} / \mathrm{Cu}(111)$ system than the structural distortions.

nderstanding and modifying materials properties for technological applications has always been a prime interest of materials science. To develop new functional materials for nanoscale electronics, it is important to accurately characterize and manipulate the quantum state of molecules adsorbed on surfaces. Organic semiconductor devices have attracted much attention due to various potential application $s^{1-5}$. Metal phthalocyanine (MPc) molecules are important for organic electronic devices because of their good chemical and thermal stability, less complicated synthesis, and tunable physical properties by substitutional flexibility ${ }^{6,7}$. They provide relatively stable chemical environments for the central metal atom ${ }^{8}$. In addition, the molecules can be employed as building blocks for a wide range of systems such as organic thin film transistors, field effect transistors, gas sensors, photovoltaic cells, and organic light emitting diodes 9 .

It is observed that the device functionalities of organic molecules can be significantly improved by controlled selective adsorption on different substrates ${ }^{10,11}$. Of course, the electronic properties of the molecules are modified by interaction with the substrate. In particular, charge transfer between substrate and molecule modifies the molecular band gap ${ }^{9,12,13}$. Recently, Zhang and coworkers have investigated the adsorption of MPc molecules $(\mathrm{M}=\mathrm{Fe}, \mathrm{Co}, \mathrm{Ni}, \mathrm{Cu}$, and $\mathrm{Zn})$ on $\mathrm{Au}(111)$ and have established the most stable configurations ${ }^{5}$. They report on significant differences in the electronic properties of $\mathrm{MnPc}$, FePc, and $\mathrm{CoPc}$ molecules. Baran and coworkers have presented a combined experimental and theoretical study of adsorption of $\mathrm{CoPc}, \mathrm{PbPc}$, and $\mathrm{SnPc}$ on $\mathrm{Ag}(111)^{14}$. They find a strong interaction of the central $\mathrm{Pb}$ atom with the $\mathrm{Ag}$ surface, leading to a reduction from $\mathrm{Pb}^{+2}$ to $\mathrm{Pb}^{0}$. Among the MPc molecules, $\mathrm{ZnC}_{32} \mathrm{~N}_{8} \mathrm{H}_{16}(\mathrm{ZnPc})$ has become a kind of model system for fundamental studies. It shows most of the basic features of the Pc molecule, because the influence of $\mathrm{Zn}$ on the electronic structure is small. Numerous optical and electrical applications of $\mathrm{ZnPc}$ in organic electronics have been discussed in the literature ${ }^{5,15}$.

The supposition that aromatic molecules without electronic coupling are physisorbed on metal surfaces is not acceptable for all systems. Though there is no electronic coupling and, hence, no covalent bonding in the pentacene/ $\mathrm{Cu}(100)$ system, it has been observed that the electronic structure of pentacence is modified significantly. This modification is due to the formation of mixed molecule-metal states ${ }^{16}$. Formation of interface states and dipoles is a central issue for active organometallic interfaces ${ }^{17,18}$. They determine the energy level alignment near the Fermi energy $\left(E_{F}\right)$ and thus the performance of the device. Key factors in the creation of interface states and dipoles are the distance between molecule and substrate and the transfer of charge between them ${ }^{19,20}$. The adsortion of a molecule can modify the work function of the substrate and thus the interface dipole, which determines the carrier injection barrier at organometallic interfaces ${ }^{18,22}$.

Yamane and coworkers have used a combination of the x-ray standing wave technique and angle-resolved photoemission spectroscopy for $\mathrm{ZnPc}$ on $\mathrm{Cu}(111)$ to analyze how the adsorbate configuration affects the chemical bonding at the interface and its electronic properties ${ }^{21}$. In addition, Gerlach and coworkers have shown experimentally that the adsorption distance on a $\mathrm{Cu}$ substrate is small because of the relatively low work function (as compared to $\mathrm{Ag}$ and $\mathrm{Au}$, for example), pointing to a strong interaction ${ }^{23}$. As a consequence, charge can easily be transferred between the substrate and adsorbed molecule to modify the electronic properties. Despite many experimental results on the adsorption of organic molecules on metal surfaces, an adequate theoretical description remains challenging ${ }^{24}$. 


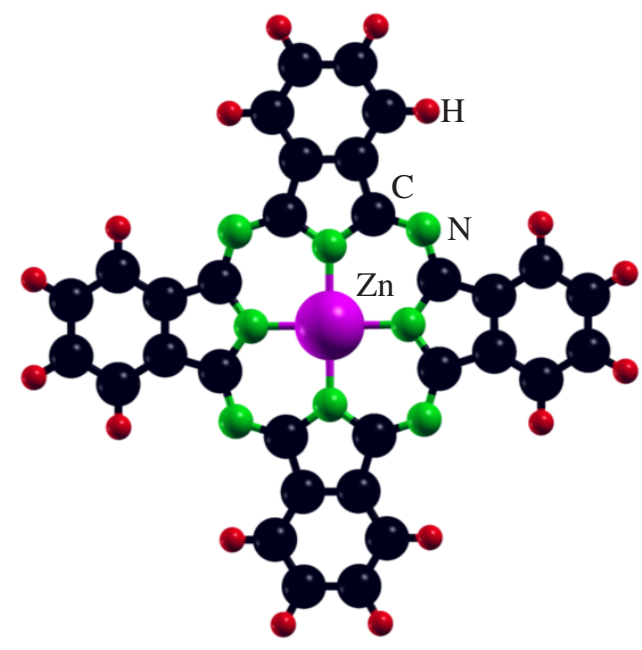

Figure $1 \mid$ Free standing $\mathrm{ZnPc}$ molecule.

In this article, we determine the adsorption geometry and electronic structure of an isolated $\mathrm{ZnPc}$ molecule on $\mathrm{Cu}(111)$. Going beyond the experiment, we are able to describe and explain the consequences of the interaction with the substrate in detail. On the one hand, we demonstrate that the Zn-N chemical bonding within the molecule is modified such that the energy levels are altered. On the other hand, a strong transfer of charge within the molecule leads to a metallic nature. This fact indicates that the charge transfer governs the properties of this organometallic interface rather than structure effects as proposed in Ref. 21.

\section{Results}

In order to investigate the adsorption behavior and electronic properties of $\mathrm{ZnPc}$ on $\mathrm{Cu}(111)$, it is necessary to first study a free standing $\mathrm{ZnPc}$ molecule. Figure 1 shows the schematic structure of the molecule, which is composed of a central $\mathrm{Zn}$ atom and four isoindole groups bridged by four additional $\mathrm{N}$ atoms, having planar D4h symmetry ${ }^{27}$. To analyze the electronic structure of the free standing $\mathrm{ZnPc}$ molecule, the total and partial DOSs are calculated and presented in Fig. 2. It is clear from the total DOS in panel (a) on the left hand side of Fig. 2, which is obtained within the GGA, that the highest occupied molecular orbital (HOMO) and lowest unoccupied molecular orbital (LUMO) are due to the Pc skeleton, with small contributions of $\mathrm{Zn}$ to the HOMO. These two highly delocalized orbitals are responsible for most of the physical properties. The state near $E_{F}$ is due to the C $2 p_{y}$ orbitals, while the states around $-2 \mathrm{eV}$ are a combination of $\mathrm{C} 2 p_{z}$, $2 p_{x}$ and N $2 p_{x}, 2 p_{z}$ orbitals. Similarly, the LUMO is due to both C $2 p_{z}$ and $\mathrm{N} 2 p_{y}$ orbitals. The group of peaks around $-4 \mathrm{eV}$ is mainly due to the C $2 p_{z}$ orbital, with small contributions of the $\mathrm{N} 2 p_{y}$ orbital. In addition, the states in the energy range from $-8 \mathrm{eV}$ to $-5.5 \mathrm{eV}$ trace back to the $\mathrm{C} 2 p_{z}, 2 p_{x}$ and $\mathrm{Zn} 3 d$ orbitals, with a very small contribution of the $\mathrm{H} 1 s$ orbital, see panels (b), (c), and (d) in Fig. 2. We observe $p d$-hybridization between the $\mathrm{C} 2 p$ and $\mathrm{Zn} 3 d$ orbitals in this energy range, where $\mathrm{Zn}$ contributes more at low energy. This fact indicates that the presence of $\mathrm{Zn}$ at the center of the molecule does not influence the basic electronic structure of the Pc skeleton, reflecting the suitability of $\mathrm{ZnPc}$ as model system for fundamental studies.

We have also calculated total and partial DOSs of a free standing $\mathrm{ZnPc}$ molecule using the hybrid functional approach (PBE0), see the right hand side of Fig. 2. The $1 \mathrm{eV}$ peak in the LUMO $\left(\mathrm{C} 2 p_{z}\right.$ and $\mathrm{N}$ $2 p_{y}$ states) is shifted to around $2 \mathrm{eV}$, while the small $\mathrm{Zn} 3 d$ peak below $E_{F}$ shifts to lower energy in the case of the hybrid approach. The $p d-$ hybridized and $\mathrm{H}$ states reveal a similar behavior. We note that our GGA DOS of free standing ZnPc shows significant improvements over previously reported results in Ref. 28, where the local density approximation has been employed, and seconds Ref. 5. The PBE0 approach yields a band gap of $1.93 \mathrm{eV}$, in excellent agreement with the experimental value ${ }^{29}$. In addition, the photoemission spectra reported in Ref. 30 are well reproduced, particularly the small feature at $-0.24 \mathrm{eV}$ which appears both in our GGA and PBE0 data. While the states found in the experiment around $-2.5 \mathrm{eV}$ could not be reproduced in previous GGA and GW calculations, they clearly reappear in our case. This difference may be related to the $\mathrm{C}$ pseudopotential, as these are $\mathrm{C}$ states.

Before discussing the electronic structure of the $\mathrm{ZnPc} / \mathrm{Cu}(111)$ system, we first address the adsorption geometry (relaxation effects). To this aim, we investigate three adsorption sites: top, bridge, and hexagonal closed packed (hcp). We also consider the angle between the wings of the $\mathrm{ZnPc}$ molecule and the substrate. Therefore, we deal with six configurations in total: top, top angle, hcp, bridge-I (B-I), bridge-II (B-II), and bridge-III (B-III). Figure 3 gives top views of
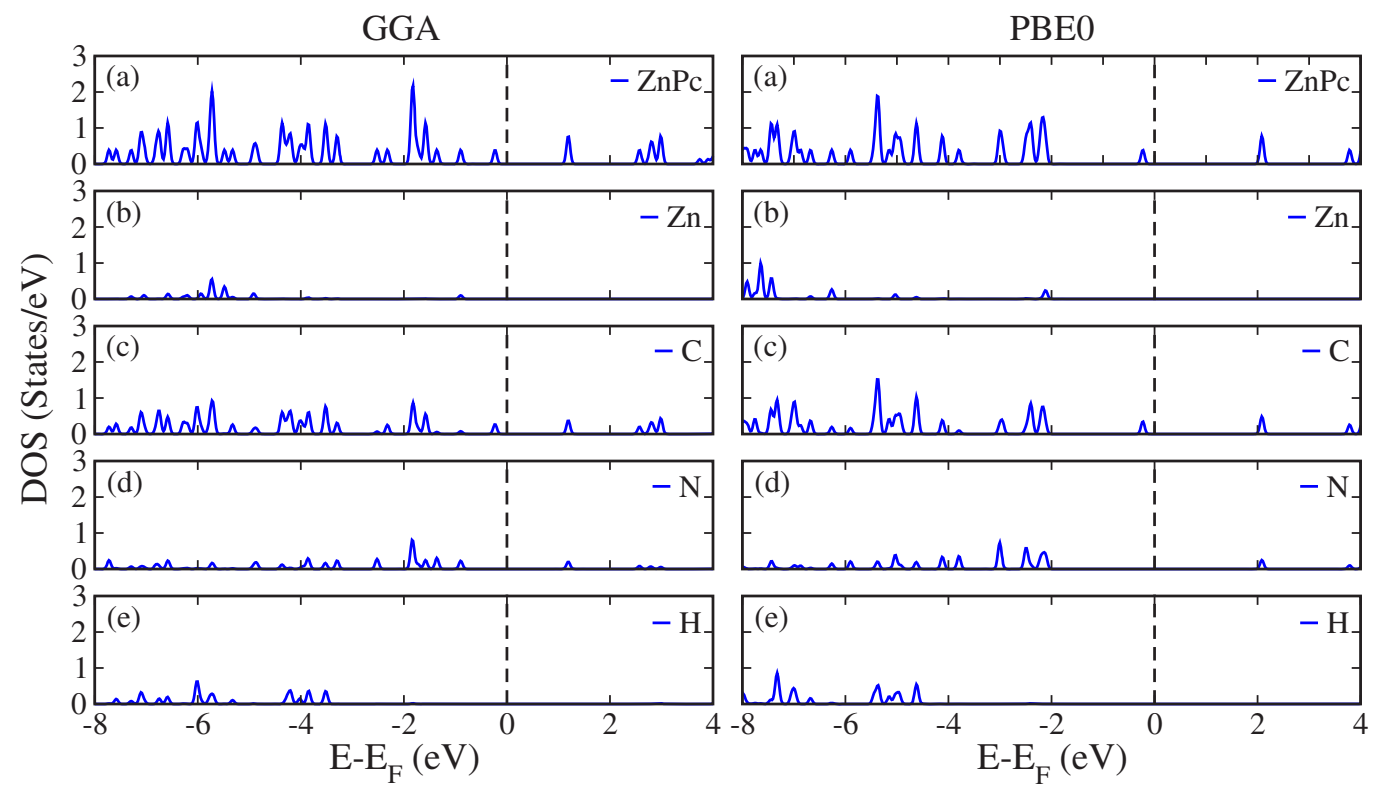

Figure $2 \mid$ Total and partial DOSs (per atom) of a free standing ZnPc molecule obtained by the (left) GGA and (right) PBE0 approaches. 


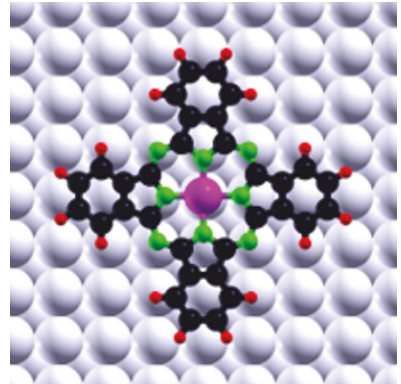

(a)

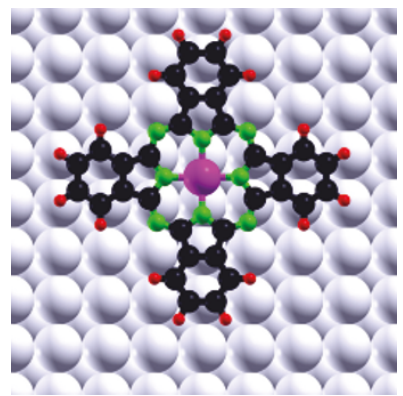

(c)

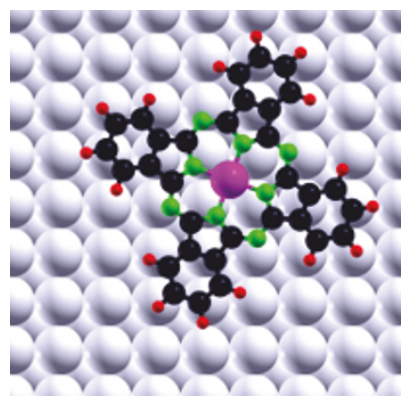

(e)

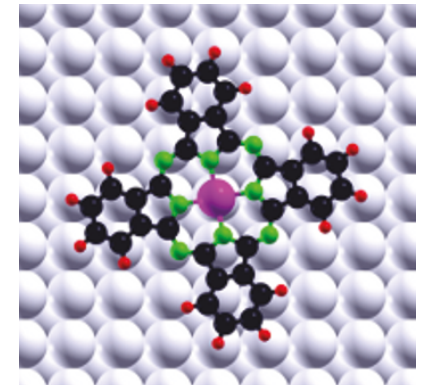

(b)

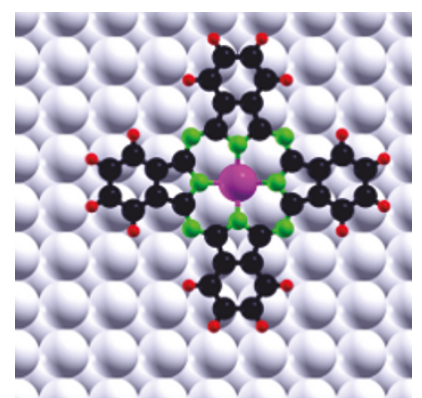

(d)

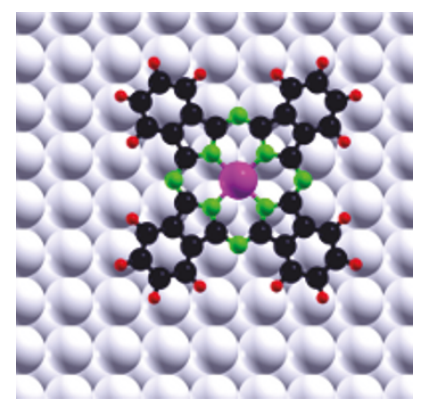

(f)
Figure 3 Adsorption configurations of $\mathrm{ZnPc}$ on $\mathrm{Cu}(111)$. (a) top, (b) top angle, (c) hcp, (d) B-I, (e) B-II, and (f) B-III.

these six configurations, which we relax with and without taking into account the vdW interaction to obtain the adsorption energy $\left(E_{a d s}\right)$. The results are given in Table 1. We find the top configuration to be most stable, in contrast to Ref. 5 where the top angle configuration was found to be favorable for the $\mathrm{ZnPc} / \mathrm{Au}(111)$ system. This difference reflects the different substrates. Higher adsorption energies for other configurations do not exclude metastable states at elevated temperatures, in particular the hcp configuration is close in energy.

After relaxing the system, we obtain a significant distortion of the $\mathrm{ZnPc}$ molecule and find substantial differences in the distances between molecule and substrate for calculations with and without $\mathrm{vdW}$ interaction. The average distances with $\mathrm{vdW}$ interaction are $3.22 \AA, 3.09 \AA, 2.98 \AA$, and $2.67 \AA$ for $\mathrm{H}, \mathrm{C}, \mathrm{N}$, and Zn, respectively.
Table I| Adsorption energies obtained for the configurations defined in Fig. 3

\begin{tabular}{ccccccc} 
& top top angle & hcp & B-I & B-II & B-III \\
\hline$E_{\text {ads }}(\mathrm{eV})$ & -0.487 & -0.381 & -0.445 & -0.223 & -0.389 & -0.241
\end{tabular}

Without vdW interaction we obtain $3.12 \AA, 2.98 \AA, 2.77 \AA$, and $2.42 \AA$, respectively. Therefore, in general, the distances are larger under inclusion of the vdW interaction, showing that the $\mathrm{vdW}$ method does not yield good results for the present system. An overestimation of the distance between an aromatic molecule and $\mathrm{Cu}$ substrate with vdW interaction has also been reported in Ref. 31 and agrees with the conjecture of Ref. 5 that the vdW method usually has drawbacks for molecule-metal interfaces. Our calculated distances obtained without vdW interaction deviate from the experimental values by $19.6 \%$ for C, $8.6 \%$ for $\mathrm{N}$, and $6.6 \%$ for $\mathrm{Zn}$. Rurali and coworkers have reported similar deviations for a perylene derivate on $\operatorname{Ag}(111)^{32}$. Toyoda and coworkers have argued that the overestimation is due to the more repulsive exchange part of the generalized gradient approximation ${ }^{33}$.

An interesting outcome of our calculations are the significant differences in the distances of the different atoms of the molecule from the substrate. They reflect a strong distortion of the $\mathrm{ZnPc}$ molecule (non-planar configuration), where the $\mathrm{Zn}$ atom is pulled towards the substrate while the Pc skeleton moves away, see Fig. 4(b). The different heights of the atoms relative to the substrate lower the symmetry upon adsorption. With vdW interaction we obtain atomic shifts of $0.31 \AA$ for $\mathrm{Zn}$ and $\mathrm{N}, 0.43 \AA$ for $\mathrm{Zn}$ and $\mathrm{C}$, and $0.55 \AA$ for $\mathrm{Zn}$ and $\mathrm{H}$, while without vdW interaction we have $0.36 \AA$ for $\mathrm{Zn}$ and $\mathrm{N}$, $0.56 \AA$ for $\mathrm{Zn}$ and $\mathrm{C}$, and $0.71 \AA$ for $\mathrm{Zn}$ and $\mathrm{H}$. These values indicate that the molecular distortions are underestimated when the $\mathrm{vdW}$ interaction is included ${ }^{21}$. An increased molecule-substrate distance and flattened molecule, see Fig. 4(c), correspond to an underestimated interface dipole. Without vdW interaction, our results show that mainly the $\mathrm{Zn}-\mathrm{N}$ bond is modified by the interaction with the substrate. As compared to the free standing molecule, the $\mathrm{Zn}-\mathrm{N}$ bond length increases from $2.00 \AA$ to $2.02 \AA$ and the N-Zn-N bond angle is heavily reduced from $180^{\circ}$ to $163^{\circ}$. The fact that a distortion alters the interface dipole can be problematic for active organometallic interfaces. To counteract the effect, fluorination of the molecule is expected to reduce the vertical distance and consequently the molecular distortion.

To understand the electronic structure of the $\mathrm{ZnPc} / \mathrm{Cu}(111)$ system, we evaluate the total and partial DOSs for the most stable configurations, see Fig. 5, and compare with the free standing molecule (Fig. 2). Due to the interaction with the $\mathrm{Cu}$ substrate, the electronic states near $E_{F}$ change significantly and the band gap disappears. Hence, the $\mathrm{ZnPc}$ molecule shows a metallic behavior, see panels (a) in Fig. 5. It is clear from panels (b) in Fig. 5 that the $\mathrm{Zn}$ states shift to lower energy by about $1 \mathrm{eV}$ as compared to the free standing molecule and therefore play no longer a role near $E_{F}$. Panels (c) and (d) in Fig. 5 show that the C $2 p_{z}$ and N $2 p_{z}$ states around $E_{F}$ are suppressed and also shift to lower energy. In the energy range from $-8 \mathrm{eV}$ to $-6 \mathrm{eV}$ we mainly find hybridized $\mathrm{Zn} 3 d$ and $\mathrm{C} 2 p$ states ( $p d$-hybridization), with small contributions of the $\mathrm{N} 2 p_{y}$ and $2 p_{z}$

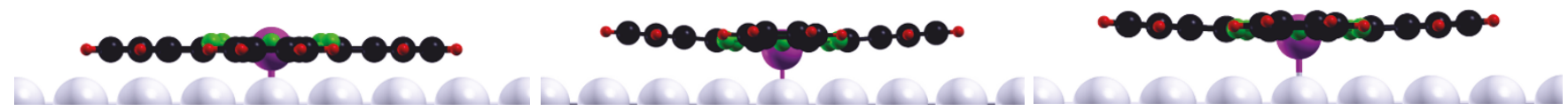

(a)

(b)

(c)

Figure $4 \mid$ Structure of $\mathrm{ZnPc}$ on $\mathrm{Cu}(111)$. (a) before relaxation, (b) after relaxation without vdW interaction, and (c) after relaxation with vdW interaction. 
GGA without vdW

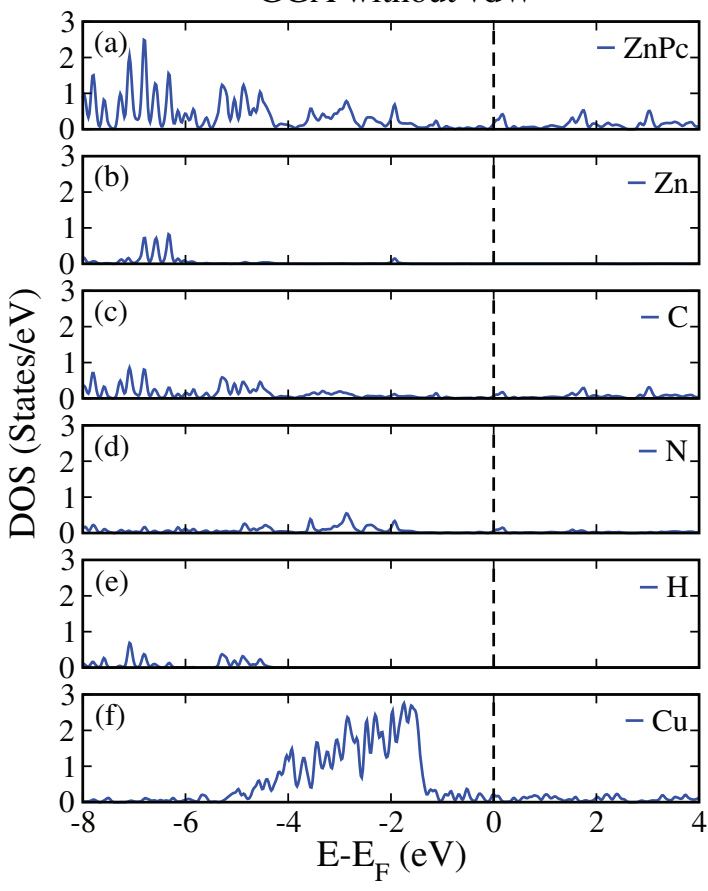

GGA with vdW
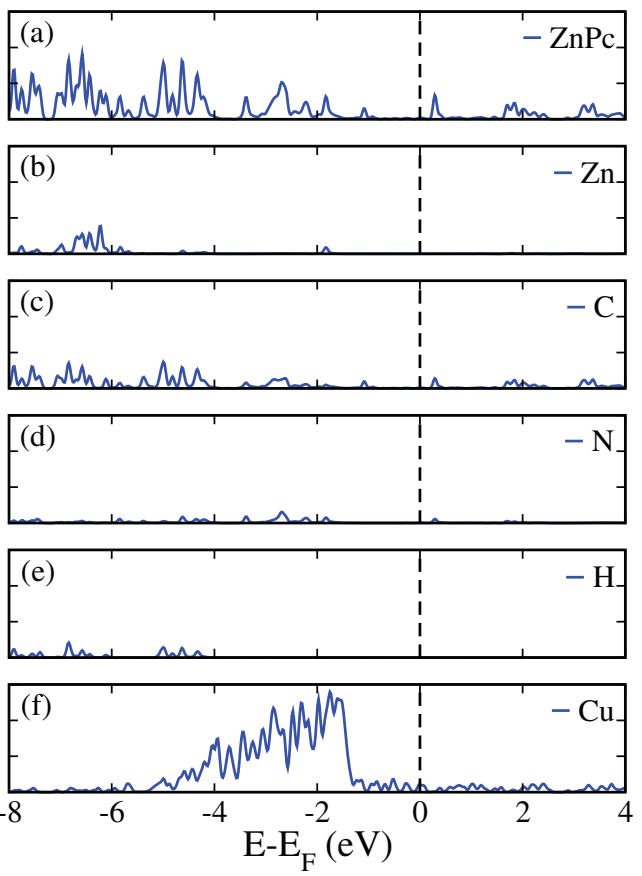

Figure $5 \mid$ (a) ZnPc, (b) Zn, (c) C, (d) N, (e) H, and (f) Cu partial DOSs (per atom) of ZnPc/Cu(111) obtained by the (left) GGA without vdW and (right) GGA with vdW approaches.

states, which also shift to lower energy as compared to the free standing molecule.

The most significant changes in the charge distribution after adsorption can be described as charge transfer from the $\mathrm{Cu}$ surface to the region between molecule and substrate, which comes along with a significant modification of the dipole perpendicular to the $\mathrm{Cu}$ surface. As a consequence of additional charge transfer within the $\mathrm{ZnPc}$ molecule, the LUMO becomes partially occupied, giving rise to a metallic nature. A similar behavior has been reported by Amsalem and coworkers ${ }^{9}$ for $\mathrm{ZnPc}$ on $\mathrm{Ag}(110)$, which we explain by the fact that the work function of $\mathrm{Ag}(4.73 \mathrm{eV})$ is similar to that of $\mathrm{Cu}$ $(4.7 \mathrm{eV})$. A metallic state is also in agreement with previous calculations for pentacene ${ }^{16}$ and benzene $e^{34}$ on a $\mathrm{Cu}$ substrate. In general, partial occupation of orbitals in MPc systems can lead to extraordinary electronic properties, including superconductivity ${ }^{9}$. Such modifications are not possible by simple physisorption of the molecule on the substrate.

Figure 5 shows that $\mathrm{Cu}$ dominates the DOS in the energy range from $-5.2 \mathrm{eV}$ to $E_{F}$, compare panles (c), (d), and (f). The peaks below $-1.8 \mathrm{eV}$ seen in panels (a) also appear in the HOMO of the

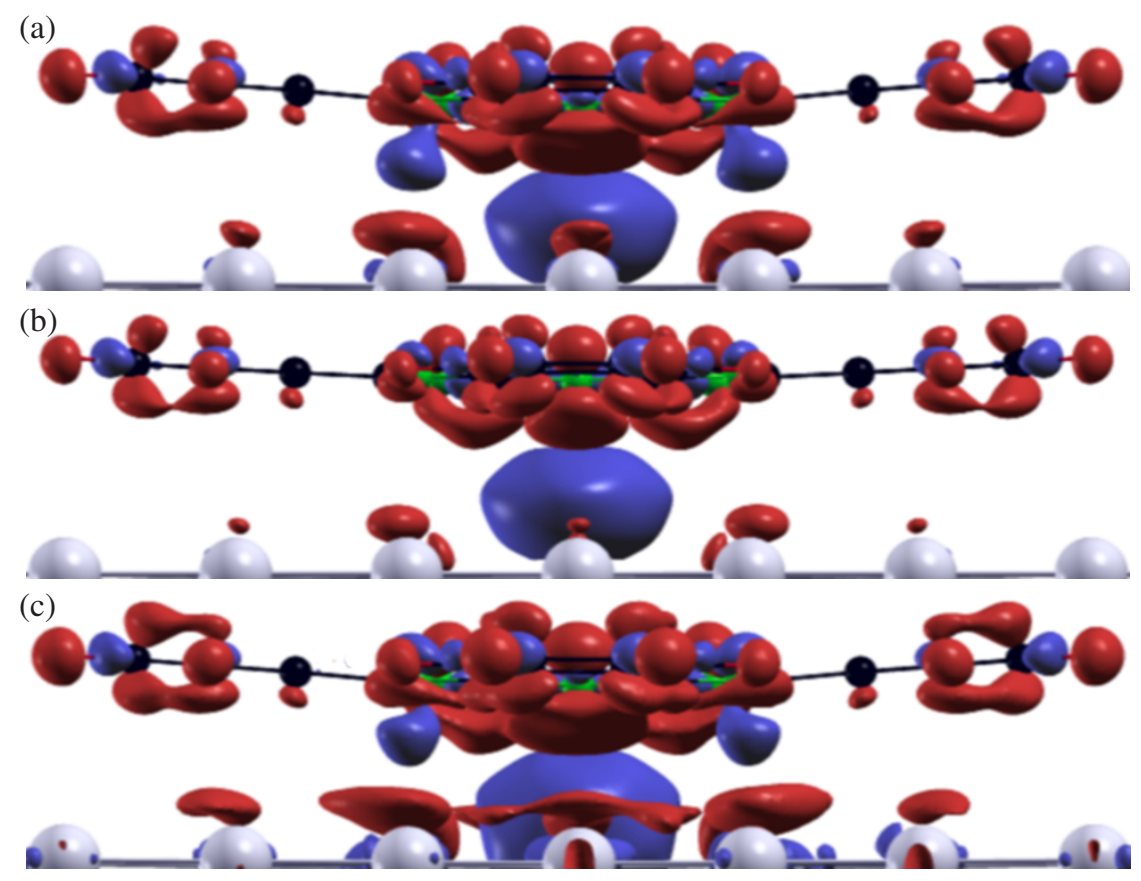

Figure 6 Charge density difference isosurfaces of $\mathrm{ZnPc} / \mathrm{Cu}(111)$ obtained by the (a) GGA without vdW, (b) GGA with vdW, and (c) PBE0 approaches. Red and blue colors represent charge depletion and accumulation, respectively. The isovalue is set to $\pm 0.005 \AA^{-3}$. 
photoemission spectra of Ref. 21. On the other hand, the small peaks near $E_{F}$ are Shockley surface states, in agreement with the experimental situation. In general, interface states determine the charge transfer and give rise to a complex electronic behavior. In order to analyze the effects on the electronic properties, we compare results without and including the vdW interaction in the two panels of Fig. 5. Switching on the vdW interaction hardly modifies the electronic states. Only the C and N 2py interface states are slightly shifted to higher energy, while the Cu DOS stays essentially the same.

To visualize the charge transfer, we show in Fig. 6 the charge density difference $\Delta \rho=\rho_{\mathrm{ZnPc} / \mathrm{Cu}(111)}-\rho_{\mathrm{ZnPc}}-\rho_{\mathrm{Cu}(111)}$, where $\rho_{\mathrm{ZnPc} / \mathrm{Cu}(111)}, \rho_{\mathrm{ZnPc}}$ and $\rho_{\mathrm{Cu}(111)}$ are the charge densities of the full system, the isolated $\mathrm{ZnPc}$ molecule, and the $\mathrm{Cu}$ substrate, respectively. In Fig. 6 results are given for GGA calculations without and with vdW interaction as well as for the PBE0 approach. The regions of charge accumulation (isovalue +0.003 ) are displayed in blue color, the regions of electron depletion (isovalue -0.003 ) in red color. We observe a significant variation of the charge density at the interface due to the adsorption of the molecule. A strong charge accumulation is found just below the center of the molecule, while regions of depletion appear both on the lower side of the molecule (facing the substrate) and on the surface of the $\mathrm{Cu}$ substrate. The charge accumulation at the interface is a consequence of the Pauli repulsion ${ }^{35,36}$. A quantitative analysis demonstrates that about 0.09 electrons are transfered into the interstitial region, while about 0.03 electrons are displaced within the $\mathrm{ZnPc}$ molecule, essentially from the outer regions towards the $\mathrm{Zn}$ atom. This redistribution of charge is responsible for the formation of a dipole pointing from the substrate to the molecule. Under inclusion of the vdW interaction the calculated charge transfer is almost the same as without vdW interaction, compare panels (a) and (b) of Fig. 6. Only the magnitude is slightly smaller, due to the overestimation of the distance between substrate and molecule. Although the vdW interaction affects this distance, we find little influence on the charge transfer and, hence, on the electronic structure. Results from PBE0 hybrid calculations are shown in panel (c) of Fig. 6. While we observe slightly more charge redistribution within the substrate, as compared to the GGA results in panels (a) and (b), the pictures are similar otherwise. We therefore can conclude that in the present system the GGA gives reliable results for the charge transfer.

\section{Discussion}

Density functional theory has been used to investigate the adsorption geometry and electronic properties of a $\mathrm{ZnPc}$ molecule on $\mathrm{Cu}(111)$. It is found that the most stable adsorption configuration is characterized by minimization of the $\mathrm{Zn}$-Cu distance. Our structural optimizations leave no doubt that the $\mathrm{Zn}$ atom is pulled to the substrate, while the Pc skeleton moves away. We have demonstrated that inclusion of the vdW interaction does not improve the relaxation results for the present system. Modification of the chemical bonding (mainly the $\mathrm{Zn}-\mathrm{N}$ bonds) alters the energy levels of the $\mathrm{ZnPc}$ molecule. It turns out that charge transfer within the $\mathrm{ZnPc}$ molecule partially fills the LUMO and induces metallic states. Most importantly, a shift of charge from the $\mathrm{Cu}(111)$ substrate and the $\mathrm{ZnPc}$ molecule into the region between them is observed. Since this will have strong effects on the interface dipole, the charge transfer governs the properties of this organometallic interface rather than the structural effects proposed in Ref. 21. Hybrid calculations result in a similar amount of charge transfer as obtained within the GGA.

\section{Methods}

The calculations are performed in the framework of density functional theory and the generalized gradient approximation (GGA) using the Vienna Ab-initio Simulation Package $^{25}$. Exchange correlation effects are treated by the Perdew-Wang functional ${ }^{26}$. For comparison, the hybrid functional approach in the PBE0 flavor is employed. In order to determine the electronic properties of a free standing $\mathrm{ZnPc}$ molecule, a geometrical optimization is performed in a $24 \AA \times 24 \AA \times 7 \AA$ supercell, where the molecule is located in the $x y$-plane. Both the free standing molecule and the $\mathrm{ZnPc} /$ $\mathrm{Cu}(111)$ system are treated by a full structural optimization until all the residual forces on the atoms are less than $0.02 \mathrm{eV} / \mathrm{A}$. We report results obtained both without and under inclusion of the van der Waals (vdW) interaction. In the $\mathrm{ZnPc} / \mathrm{Cu}(111)$ supercell, the molecule sits on a Cu(111) substrate of $9 \AA$ thickness and is followed by a $16 \AA$ thick vacuum layer to avoid artificial interaction in the slab model. Our final $\mathrm{ZnPc} / \mathrm{Cu}(111)$ system comprises 543 atoms (57 of $\mathrm{ZnPc}$ and 486 of $\mathrm{Cu}$ ). Gamma point sampling is employed and the electronic wave function is expanded in plane waves with a kinetic energy cutoff of $400 \mathrm{eV}$. Moreover, a Gaussian smearing with a width of $0.05 \mathrm{eV}$ is implemented.

1. Eremtchenko, M., Schaefer, J. A. \& Tautz, F. S. Understanding and tuning the epitaxy of large aromatic adsorbates by molecular design. Nature 425, 602-605 (2003).

2. Barth, J. V., Costantini, G. \& Kern, K. Engineering atomic and molecular nanostructures at surfaces. Nature 437, 671-679 (2005).

3. Du, S. X. et al. Selective nontemplated adsorption of organic molecules on nanofacets and the role of bonding patterns. Phys. Rev. Lett. 97, 156105 (2006).

4. Gao, L. et al. Constructing an array of anchored single-molecule rotors on gold surfaces. Phys. Rev. Lett. 101, 197209 (2008).

5. Zhang, Y. Y., Du, S. X. \& Gao, H. J. Binding configuration, electronic structure, and magnetic properties of metal phthalocyanines on a $\mathrm{Au}(111)$ surface studied with ab initio calculations. Phys. Rev. B 84, 125446 (2011).

6. Hains, A. W., Liang, Z., Woodhouse, M. A. \& Gregg, B. A. Molecular semiconductors in organic photovoltaic cells. Chem. Rev. 110, 6689-6735 (2010).

7. Yu, S. et al. Inhomogeneous charge transfer within monolayer zinc phthalocyanine absorbed on $\mathrm{TiO}_{2}(110)$. J. Chem. Phys. 136, 154703 (2012)

8. Li, Z., Li, B., Yang, J. \& Hou, J. G. Single-molecule chemistry of metal phthalocyanine on noble metal surfaces. Acc. Chem. Res. 43, 954-962 (2010).

9. Amsalem, P., Giovanelli, L., Themlin, J. M. \& Angot, T. Electronic and vibrational properties at the $\mathrm{ZnPc} / \mathrm{Ag}(110)$ interface. Phys. Rev. B 79, 235426 (2009).

10. Salem, A. K., Searson, P. C. \& Leong, K. W. Multifunctional nanorods for gene delivery. Nat. Mater. 2, 668-671 (2003).

11. Germishuizen, W. A. et al. Selective dielectrophoretic manipulation of surfaceimmobilized DNA molecules. Nanotechnology 14, 896 (2003).

12. Wang, L. L. \& Cheng, H. P. Density functional study of the adsorption of a $\mathrm{C}_{60}$ monolayer on $\mathrm{Ag}(111)$ and $\mathrm{Au}(111)$ surfaces. Phys. Rev. B 69, 165417 (2004).

13. Filibian, M. et al. Strong electronic correlations in $\mathrm{Li}_{x} \mathrm{ZnPc}$ organic metals. Phys. Rev. Lett. 100, 117601 (2008).

14. Baran, J. D. et al. Theoretical and experimental comparison of $\mathrm{SnPc}, \mathrm{PbPc}$, and CoPc adsorption on Ag(111). Phys. Rev. B 81, 075413 (2010).

15. Schünemann, C. et al. Zinc phthalocyanine - Influence of substrate temperature, film thickness, and kind of substrate on the morphology. Thin Solid Films 519, 3939-3945 (2011).

16. Ferretti, A. et al. Mixing of electronic states in pentacene adsorption on copper Phys. Rev. Lett. 99, 046802 (2007).

17. Ishii, H., Sugiyama, K., Ito, E. \& Seki, K. Energy level alignment and interfacial electronic structures at organic/metal and organic/organic interfaces. Adv. Mater. 11, 605-625 (1999)

18. Hwang, J., Wan, A. \& Kahn, A. Energetics of metalorganic interfaces: New experiments and assessment of the field. Mater. Sci. Eng. R 64, 1-31 (2009)

19. Morikawa, Y., Ishii, H. \& Seki, K. Theoretical study of $n$-alkane adsorption on metal surfaces. Phys. Rev. B 69, 041403(R) (2004).

20. Vázquez, H., Dappe, Y. J., Ortega, J. \& Flores, F. Energy level alignment at metal/ organic semiconductor interfaces: "Pillow" effect, induced density of interface states, and charge neutrality level. J. Chem. Phys. 126, 144703 (2007).

21. Yamane, H. et al. Site-specific geometric and electronic relaxations at organicmetal interfaces. Phys. Rev. Lett. 105, 046103 (2010).

22. Bagus, P. S., Staemmler, V. \& Wöll, C. Exchangelike effects for closed-shell adsorbates: Interface dipole and work function. Phys. Rev. Lett. 89, 096104 (2002).

23. Gerlach, A., Sellner, S., Schreiber, F., Koch, N. \& Zegenhagen, J. Substratedependent bonding distances of PTCDA: A comparative x-ray standing-wave study on $\mathrm{Cu}(111)$ and $\mathrm{Ag}(111)$. Phys. Rev. B 75, 045401 (2007).

24. Koch, N. et al. Adsorption-induced intramolecular dipole: Correlating molecular conformation and interface electronic structure. J. Am. Chem. Soc. 130, 7300-7304 (2008).

25. Kresse, G. \& Furthmüller, J. Efficient iterative schemes for ab initio total-energy calculations using a plane-wave basis set. Phys. Rev. B 54, 11169-11186 (1996).

26. Perdew, J. P. et al. Atoms, molecules, solids, and surfaces: Applications of the generalized gradient approximation for exchange and correlation. Phys. Rev. B 46, 6671-6687 (1992).

27. Shen, X. et al. Spin filter effect of manganese phthalocyanine contacted with single-walled carbon nanotube electrodes. J. Chem. Phys. 132, 054703 (2010).

28. Senthilarasu, S., Sathyamoorthy, X. R., Ascencio, J. A., Lee, S. H. \& Hahn, Y. B. Dielectric and ac conduction properties of zinc phthalocyanine $(\mathrm{ZnPc})$ thin films. J. Appl. Phys. 101, 034111 (2007).

29. Gao, W. \& Kahn, A. Electronic structure and current injection in zinc phthalocyanine doped with tetrafluorotetracyanoquinodimethane: Interface versus bulk effects. Org. Electron. 3, 53-63 (2002). 
30. Umari, P. \& Fabris, S. Importance of semicore states in GW calculations for simulating accurately the photoemission spectra of metal phthalocyanine molecules. J. Chem. Phys. 136, 174310 (2012).

31. Berland, K., Einstein, T. L. \& Hyldgaard, P. Rings sliding on a honeycomb network: Adsorption contours, interactions, and assembly of benzene on $\mathrm{Cu}(111)$. Phys. Rev. B 80, 155431 (2009).

32. Rurali, R., Lorente, N. \& Ordejon, P. Comment on "Molecular Distortions and Chemical Bonding of a Large $\pi$-Conjugated Molecule on a Metal Surface" Phys. Rev. Lett. 95, 209601 (2005).

33. Toyoda, K. et al. First-principles study of the pentacene/Cu(111) interface: Adsorption states and vacuum level shifts. J. Electron Spectrosc. Relat. Phenom. 174, 78-84 (2009).

34. Lorente, N., Hedouin, M. F. G., Palmer, R. E. \& Persson, M. Chemisorption of benzene and STM dehydrogenation products on $\mathrm{Cu}(100)$. Phys. Rev. B 68, 155401 (2003).

35. Rojas, G. et al. Surface state engineering of molecule-molecule interactions. Phys. Chem. Chem. Phys. 14, 4971-4976 (2012).
36. Rusu, P. C., Giovannetti, G., Weijtens, C., Coehoorn, R. \& Brocks, G. Firstprinciples study of the dipole layer formation at metal-organic interfaces. Phys. Rev. B 81, 125403 (2010).

\section{Author contributions}

B.A. and S.N. performed the calculations. All authors wrote the manuscript.

\section{Additional information}

Competing financial interests: The authors declare no competing financial interests. License: This work is licensed under a Creative Commons

Attribution-NonCommercial-NoDerivs 3.0 Unported License. To view a copy of this license, visit http://creativecommons.org/licenses/by-nc-nd/3.0/

How to cite this article: Amin, B., Nazir, S. \& Schwingenschlögl, U. Molecular distortion and charge transfer effects in $\mathrm{ZnPc} / \mathrm{Cu}(111)$. Sci. Rep. 3, 1705; DOI:10.1038/srep01705 (2013). 